\title{
NOVEL IMAGE-DEPENDENT QUALITY ASSESSMENT MEASURES
}

\author{
${ }^{1}$ Asaad Noori Hashim and ${ }^{2}$ Zahir M. Hussain \\ ${ }^{1}$ Department of Computer Science, University of Babylon, P.O. Box 4, Babylon, Iraq \\ ${ }^{2}$ Department of Computer Science, University of Kufa, P.O. Box 21, Kufa, Najaf, and \\ Adjunct Professor, RMIT, Vic.3000, Australia
}

Received 2013-12-11; Revised 2014-01-06; Accepted 2014-04-07

\begin{abstract}
The image is a 2D signal whose pixels are highly correlated in a 2D manner. Hence, using pixel by pixel error what we called previously Mean-Square Error, (MSE) is not an efficient way to compare two similar images (e.g., an original image and a compressed version of it). Due to this correlation, image comparison needs a correlative quality measure. It is clear that correlation between two signals gives an idea about the relation between samples of the two signals. Generally speaking, correlation is a measure of similarity between the two signals. An important step in image similarity was introduced by Wang and Bovik where a structural similarity measure has been designed and called SSIM. The similarity measure SSIM has been widely used. It is based on statistical similarity between the two images. However, SSIM can produce confusing results in some cases where it may give a non-trivial amount of similarity while the two images are quite different. This study proposes methods to determine a reliable similarity between any two images, similar or dissimilar, in the sense that dissimilar images have near-zero similarity measure, while similar images give near-one (maximum) similarity. The proposed methods are based on image-dependent properties, specifically the outcomes of edge detection and segmentation, in addition to the statistical properties. The proposed methods are tested under Gaussian noise, impulse noise and blur, where good results have been obtained even under low Peak Signal-to-Noise Ratios (PSNR's).
\end{abstract}

Keywords: Image Structural Similarity, Edge Detection, Image Segmentation, Image Processing

\section{INTRODUCTION}

An important feature of natural images is that they are highly structured signals, meaning that the image samples exhibit strong correlation; this is more evident when samples are in spatial proximatity. This $2 \mathrm{D}$ correlation carries important information about the structure of the objects in the image.

An objective image quality measure can have a significant role in image processing and its applications, where it can be used to monitor and adjust image quality. Also, a quality measure can be used to optimize algorithms and parameter settings of image processing systems, an to benchmark image processing algorithms. Machine evaluation of image and video quality is important for many image processing systems, for Corresponding Author: Z.M. Hussain, Department of Computer Science, University of Kufa, P.O. Box 21, Kufa, Najaf, Iraq. example, systems used for compression, restoration, enhancement, etc. The goal of quality assessment is to find robust techniques for objective evaluation of image quality in accord with subjective human assessment.

Over the years many researchers have contributed to the design and implementation of reference quality assessment algorithms. Wang and Bovik (2002) avoided using traditional mean-squared error methods and proposed a model for any image distortion that is dependent on a distortion in a combination of three quantities: Correlation, luminance and contrast.

Wang et al. (2004) proposed a promising technique (SSIM) for distance covariance to measuring the structural similarity based on number of statistical measurements such as mean, standard deviation and they produced a new relation among these standards Equation 1: 


$$
\rho(x, y)=\frac{\left(2 \mu_{x} \mu_{y}+C_{1}\right)\left(2 \sigma_{x y}+C_{2}\right)}{\left(\mu_{x}^{2}+\mu_{y}^{2}+C_{1}\right)\left(\sigma_{x}^{2}+\sigma_{y}^{2}+C_{2}\right)}
$$

where, $\rho(x, y)$ is the SSIM measure between two images $\mathrm{x}$ and $\mathrm{y}, \mu_{\mathrm{x}}$ and $\sigma_{\mathrm{x}}^{2}$ are the statistical mean and variance of pixels in image $x$ ( $\mu y, \sigma_{x}^{2}$ are defined similarly) $\sigma_{x y}$ is the statistical variance between pixels in images $x$ and $y$ while the constants $C_{1}$ and $C_{2}$ are defined as $C_{1}=\left(K_{1} L\right)^{2}$ and $\mathrm{C}_{2}=\left(\mathrm{K}_{2} \mathrm{~L}\right)^{2}$, with $\mathrm{K}_{1}$ and $\mathrm{K}_{2}$ are small constants and $\mathrm{L}=255$ (maximum pixel value).

This approach gives high level of similarity for noise free condition while it goes to zero when noise increase, in other words it gives similarity with two different images due to it dependent only the statistics features of images which may have some correlations. SSIM can't reveal all image structural properties, so we need to more specific measurements that are image-dependent.

Sheikh et al. (2006) presented results of an extensive subjective quality assessment. In their study a number of distorted images were evaluated by a number of human subjects, where image quality data obtained from human quality judgments is used to evaluate several full reference image quality assessment methods. This study was the largest subjective image quality study in the literature in terms of number of images, distortion types and the number of human evaluations.

A recent improvement on SSIM is presented by Sampat et al. (2009): The Complex Wavelet SSIM (CWSSIM). It is based on wavelet coefficients that are extracted at the same spatial locations in the same wavelet subbands of the two images under test. This approach is shown to be less sensitive to small geometric variations or distortions (such as rotations, translations and difference in scale).

Szekely et al. (2007) improved similarity testing by adding a new distance measurement called "Energy Statistics" based on the following formula:

$$
\mathrm{D}(\mu, \mathrm{v})=2 \varepsilon[\mathrm{d}(\mathrm{X}, \mathrm{Y})]-\varepsilon\left[\mathrm{d}\left(\mathrm{X}, \mathrm{X}^{\prime}\right]-\varepsilon\left[\mathrm{d}\left(\mathrm{Y}, \mathrm{Y}^{\prime}\right]\right)\right)
$$

where, $\varepsilon$ is the expectation and $\mathrm{d}(\mathrm{X}, \mathrm{Y})$ is the Euclidean distance. This measure considers statistical observation and statistical potential energy. Energy statistics is a function of distance between statistical observations. This approach has a high rate of complexities and computational difficulties.

Reference Zhang et al. (2009) explains many limitations and challenges of current approached of image quality measurement. It is stated that each kind of image difference will cause a different kind of distortion in perceptual visual domain. Generally, these changes include:

- Scale, orientation, lighting and image contrast.

- Spatial distribution of texture

- Position of objects

Some kinds of distortion may higly affect the image, even if distortion is small, for example:

- Sharpness of image contours

- Other distortions or artifacts in sensitive regions like the face

Kaur et al. (2012) improved the performance of metrics like Coefficient of Correlation (CoC) and Structural Similarity Index (SSIM) for image recognition in real-time environment. $\mathrm{Li}$ et al. (2010) used a similarity assessment to select the images for synthesis, where a new similarity measure has been proposed using complex wavelets. This measure has been shown to be robust to small rotations and translations as well as large intensity and contrast changes.

Dan et al. (2010) proposed a novel image quality assessment technique which is based on the conventional SSIM and the discrete cosine transform (DCT). The method presents a frequency structural comparison by weighting the frequency components depending on the sensitivity of human eye.

Liu and Wang (2011) introduced a similarity measure based on edge structural similarity; while Liu et al. (2011) presented an objective fusion quality index.

Please note that the above-mentioned similarity measures are all based on statistical moments, on which we will focus in this study, while there are other moments that can also be used to test similarity (Lajevardi and Hussain, 2010a; 2010b).

Blasch et al. (2008) presented a novel approach on objective non-reference image fusion performance assessment. The proposed measure is an extension of the Universal Image Quality Index (UIQI); where its weighting factor is the similarity between blocks of pixels in the input images and the fused image.

In this study, we enhance the basic SSIM, proposed by Wang et al. (2004) and study the performance of SSIM and the proposed enhanced method under noisy conditions and blur. The enhancement is based on image segmentation and edge detection techniques to give more reliable similarity measure.

\section{RATIONALE}

We noticed that SSIM measure introduced by Wang et al. (2004) gives false similarity between 
unrelated images; hence, it needs more image-dependent properties to be reliable. We utilized segmentation and edge properties and combined them with SSIM to get the enhanced measure mSSIM; also we tested SSIM and Mssim under disruptive conditions like Gaussian noise, impulse noise and blur.

\section{THE PROPOSED MEASURES}

The design of SSIM was based on image statistical properties, Wang et al. (2004), hence the non-zero SSIM measure $\rho(x, y)$ between unrelated images $x$ and $\mathrm{y}$. We noticed that even straightforward segmentation (of the two images $\mathrm{x}$, $\mathrm{y}$ into K-pairs of corresponding sub-images $\left.\mathrm{x}_{\mathrm{i}}, \mathrm{y}_{\mathrm{i}}, \mathrm{i}=1,2, \ldots, \mathrm{K}\right)$ can substantially reduce the chance of statistical similarity between all available segments, therefore we propose the following image dependent measure Equation 2:

$$
\zeta(\mathrm{x}, \mathrm{y})=\prod_{\mathrm{i}=1}^{\mathrm{K}} \rho\left(\mathrm{x}_{\mathrm{i}}, \mathrm{y}_{\mathrm{i}}\right)
$$

Similarly, the inclusion of edge effects into SSIM will highly reduce the chance of statistical similarity, hence we propose the following image-dependent measure Equation 3:

$\eta(x, y)=R(x, y) \cdot \rho(x, y)$

Noting that $\mathrm{R}(\mathrm{x} ; \mathrm{y})$ is the $2 \mathrm{D}$ edge correlation coefficient defined as Equation 4:

$$
R(x, y)=\left|\frac{\Sigma_{i} \Sigma_{j}\left(g_{i j}-g_{o}\right)\left(h_{i j}-h_{o}\right)}{\sqrt{\left[\sum_{i} \Sigma_{j}\left(g_{i j}-g_{o}\right)^{2}\right]\left[\Sigma_{i} \Sigma_{j}\left(h_{i j}-h_{o}\right)^{2}\right]}}\right|
$$

where, $\mathrm{g}$ and $\mathrm{h}$ are the new images resulting from applying an edge detection technique to the test images $\mathrm{x}$ and $\mathrm{y}$, respectively, while $g_{o}$ and $h_{o}$ are their global means.

\section{THE TEST ENVIRONMENT}

The proposed SSIM measures have been tested under Gaussian noise and blur. Impulse noise, e $=[e(i, j)]$, which is a source of noise in many image processing systems, has also been considered. The arrival time of this noise process at an instant $\mathrm{k}$ is formulated as a Poisson process $b_{k}$ with parameter $\lambda$, while the amplitude of any noisy sample is formulated as a Gaussian process $g_{k}$ with zero mean and variance of $\sigma^{2}$. The overall impulsive noise process $i_{\mathrm{k}}$ is given by Al-Mawali et al. (2010) Equation 5: $\mathrm{i}_{\mathrm{k}}=\mathrm{b}_{\mathrm{k}} \cdot \mathrm{g}_{\mathrm{k}}$

If the random variable that represents the time count of arrival (since the last impulse) is $\mathrm{T}$, then the probability of arriving $\mathrm{m}$ samples after the previous impulse, $\mathrm{p}(\mathrm{m})$, will be Equation 6:

$\mathrm{p}(\mathrm{k})=\mathrm{p}(\mathrm{T}=\mathrm{k})=\exp (-\lambda) \cdot\left(\lambda^{\mathrm{k}} / \mathrm{k} !\right) ; \mathrm{k}=0,1,2, \ldots$

Noting that Equation 7:

$\varepsilon(\mathrm{T})=\operatorname{var}(\mathrm{T})=\lambda$

The power of the Gaussian amplitude $\sigma^{2}$ will contribute a total noise power of Equation 8:

$\mathrm{n}_{\mathrm{p}}=\sigma^{2} / \lambda$

Hence, we define r, the Peak Signal to Noise Ratio (PSNR), as follows Equation 9:

$\mathrm{r}=\frac{\mathrm{L}^{2}}{\mathrm{n}_{\mathrm{p}}}=\lambda \frac{\mathrm{L}^{2}}{\sigma^{2}}$

\section{RESULTS}

The proposed measures as well as SSIM have been simulated using MATLAB. Note that $0 \leq \rho(x, y) \leq 1$, so are $\zeta(\mathrm{x}, \mathrm{y})$ and $\eta(\mathrm{x}, \mathrm{y})$. For completely similar images we have $\rho(x, y)=1$; while for totally different images we have $\rho(x, y)=0$. It is better to calculate similarity measures locally not globally; hence, an $\mathrm{M} \times \mathrm{M}$ window $(\mathrm{M}=11)$ is used with a standard deviation of 1.5, Wang et al. (2004). The constants $\mathrm{C} 1=\left(\mathrm{K}_{1} \mathrm{~L}\right)^{2}$ and $\mathrm{C}_{2}=\left(\mathrm{K}_{2} \mathrm{~L}\right)^{2}\left(\mathrm{~K}_{1}\right.$ and $\mathrm{K}_{2}$ being small constants, $\left.\mathrm{L}=255\right)$ where chosen as $\mathrm{K}_{1}=0.01$ and $\mathrm{K}_{2}=0.03$, Wang et al. (2004). Note that the performance of SSIM is insensitive to these constants, Wang et al. (2004).

\subsection{Performance under Gaussian Noise}

First we implemented the Segmentation-based Measure (mSSIM) as per Equation 2 and tested its performance when the other image is corrupted with Gaussian noise. Peak Signal to Noise Ratio (PSNR) was used in this test as follows:

$$
\operatorname{PSNR}=\frac{\mathrm{L}^{2}}{\mathrm{p}_{\mathrm{n}}}
$$


where, $\mathrm{p}_{\mathrm{n}}$ is the Gaussian noise variance (power). The result of using mSSIM for two similar images is shown in Fig. 1a, with performance of mSSIM as compared to SSIM (represented by Equation 1) is

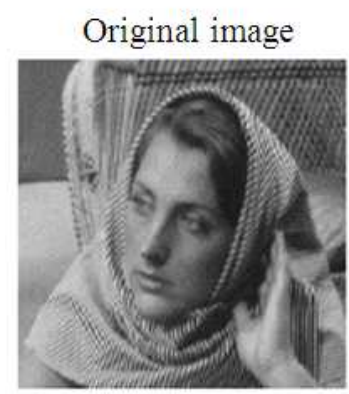

Noisy image; PSNR $(\mathrm{dB})=10$

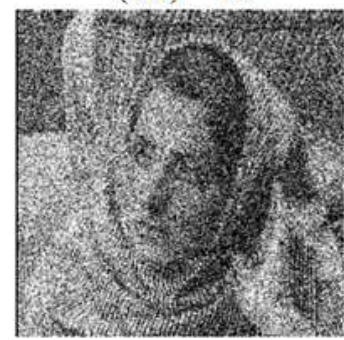

(a)

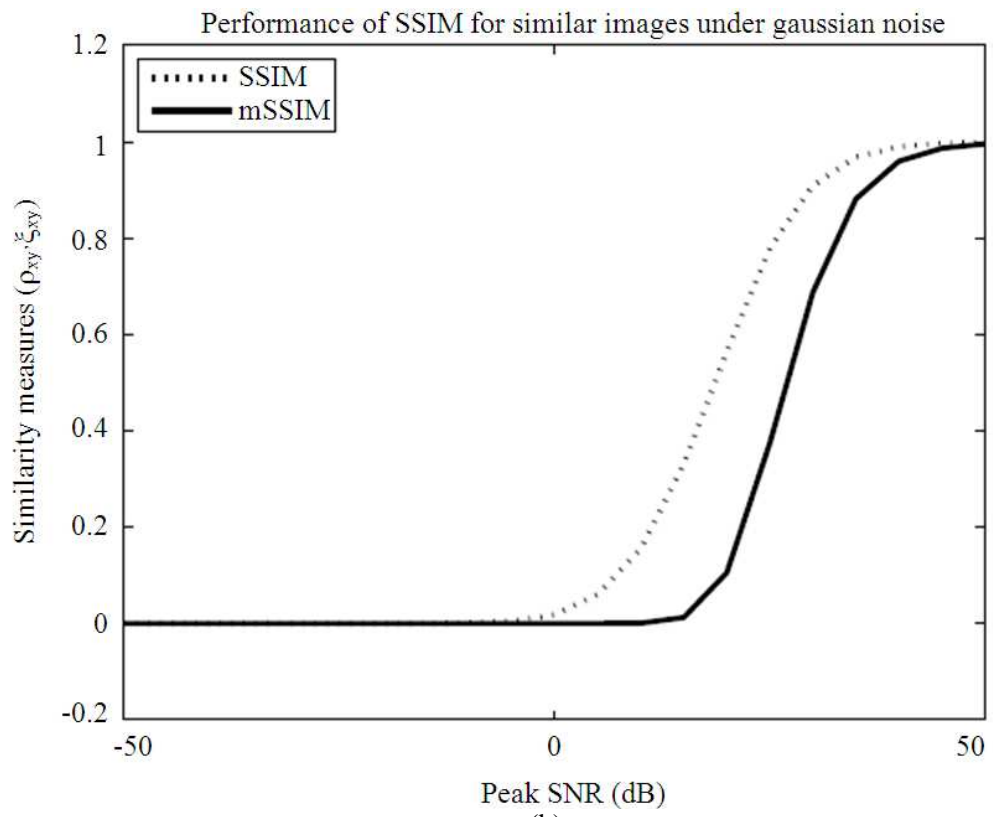

(b) "moon" from MATLAB.

Noisy image;

$$
\operatorname{PSNR}(\mathrm{dB})=50
$$

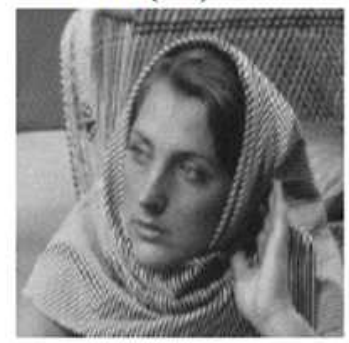

Noisy image; PSNR

$(d B)=-50$

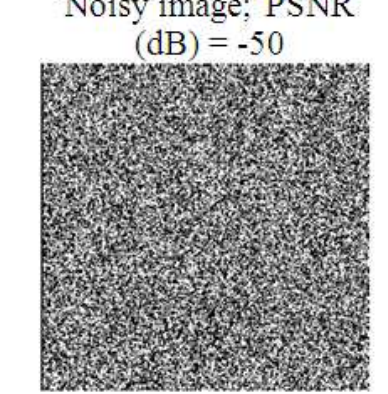

(a)

ges under gaussian noise

shown in Fig. 1b; while the result of comparing two dissimilar images is shown in Fig. $2 a$ and b, respectively. We used the images "woman" and 


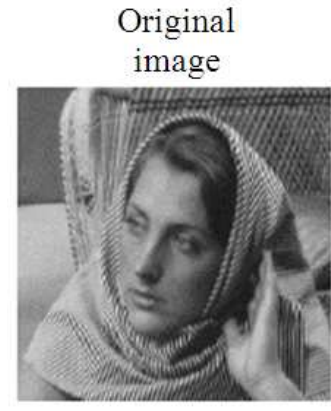

Noisy image;

$\operatorname{PSNR}(\mathrm{dB})=10$

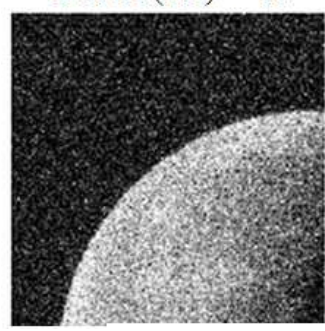

Noisy image; PSNR

$(\mathrm{dB})=50$

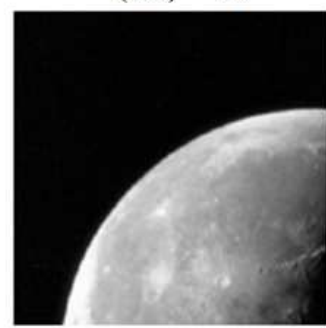

Noisy image; PSNR

$$
(\mathrm{dB})=-50
$$

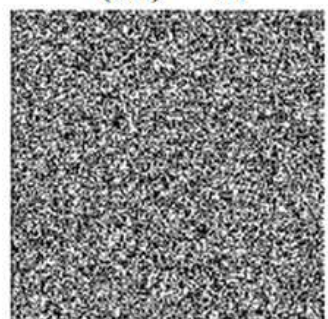

(a)

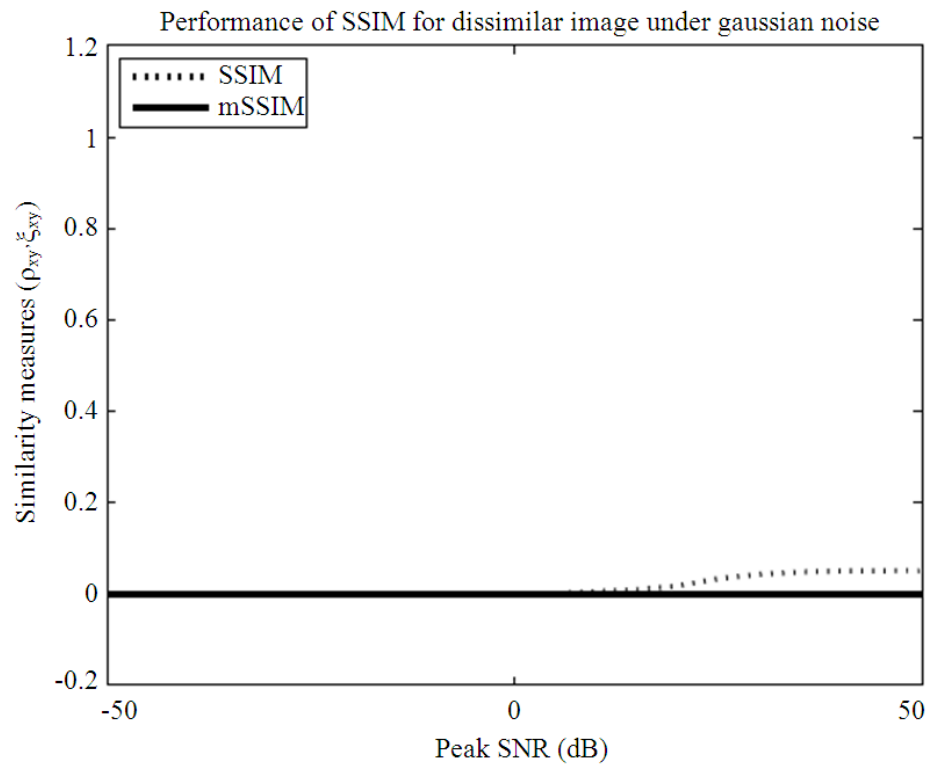

(b)

Fig. 2. Performance of SSIM and mSSIM using dissimilar images under Gaussian noise.(a) Above: The test images. (b) Below: Performance comparison between SSIM and MSSIM

Secondly, we implemented the Edge-based Measure (eSSIM) as per Equation 3 and tested its performance under Gaussian noise. Canny method has been utilized for edge detection, Canny (1986); though other methods can also be used. The results are shown in Fig. 3 and 4, with performance of eSSIM compared to that of SSIM (represented by Equation 1). In case of dissimilar images, a clearer comparison can be viewed using logarithmic scale a shown in Fig. 4. 


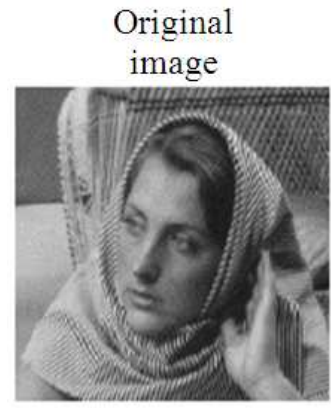

Noisy image; PSNR $(\mathrm{dB})=10$

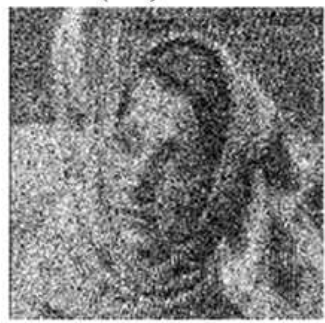

Noisy image; PSNR

$(\mathrm{dB})=50$

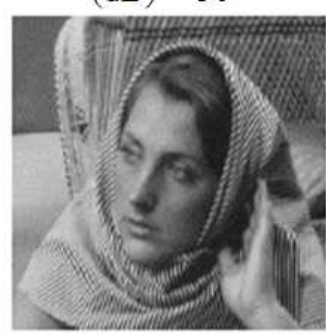

Noisy image; PSNR $(\mathrm{dB})=-50$

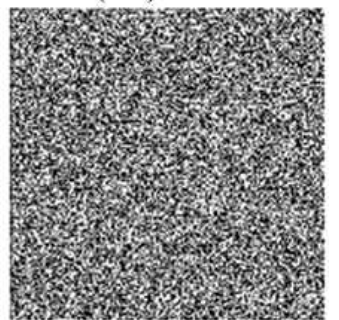

(a)

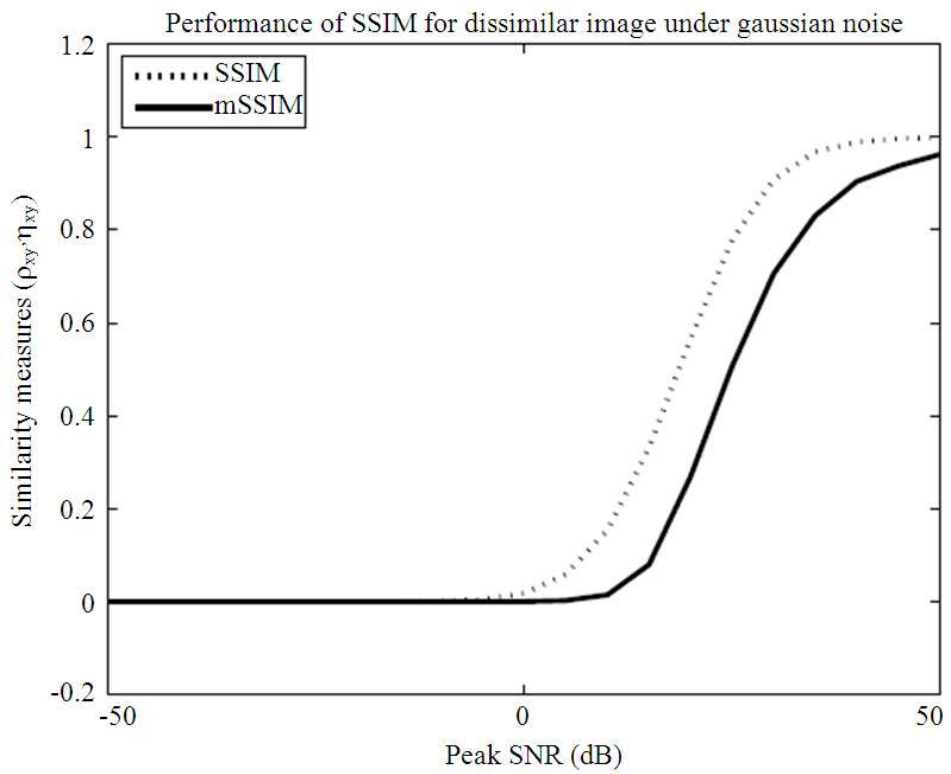

(b)

Fig. 3. Performance of SSIM and eSSIM using similar images under Gaussian noise (a) Above: The test images (b) Below: Performance comparison between SSIM and eSSIM

\subsection{Performance Under Blur}

The proposed methods have also been tested under blur. We simulated blur effect as spatial windowing (convolution) with a 2D averager, with window length W. Figure 5 and 6 show the performance of eSSIM as compared to that of SSIM under blur for different window lengths. 


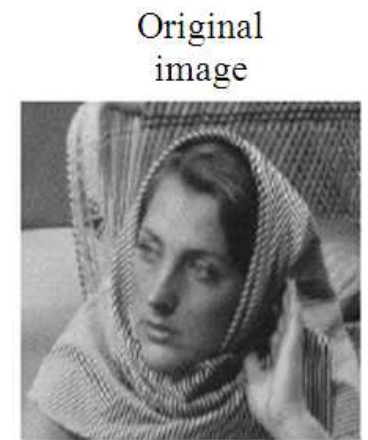

Noisy image; PSNR $(\mathrm{dB})=10$

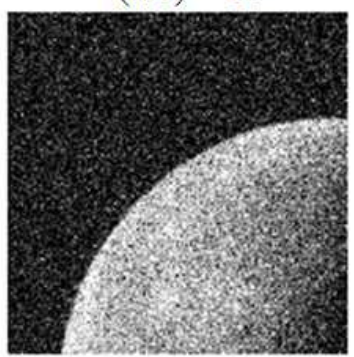

Noisy image; PSNR

$(\mathrm{dB})=50$

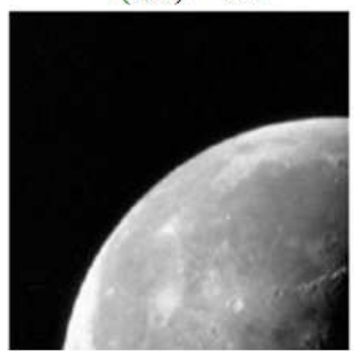

Noisy image; PSNR

$(\mathrm{dB})=-50$

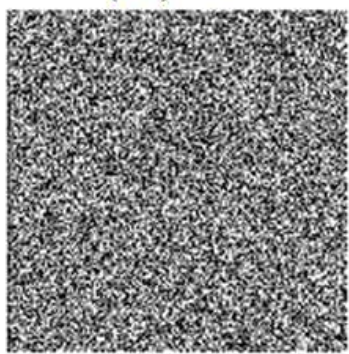

(a)

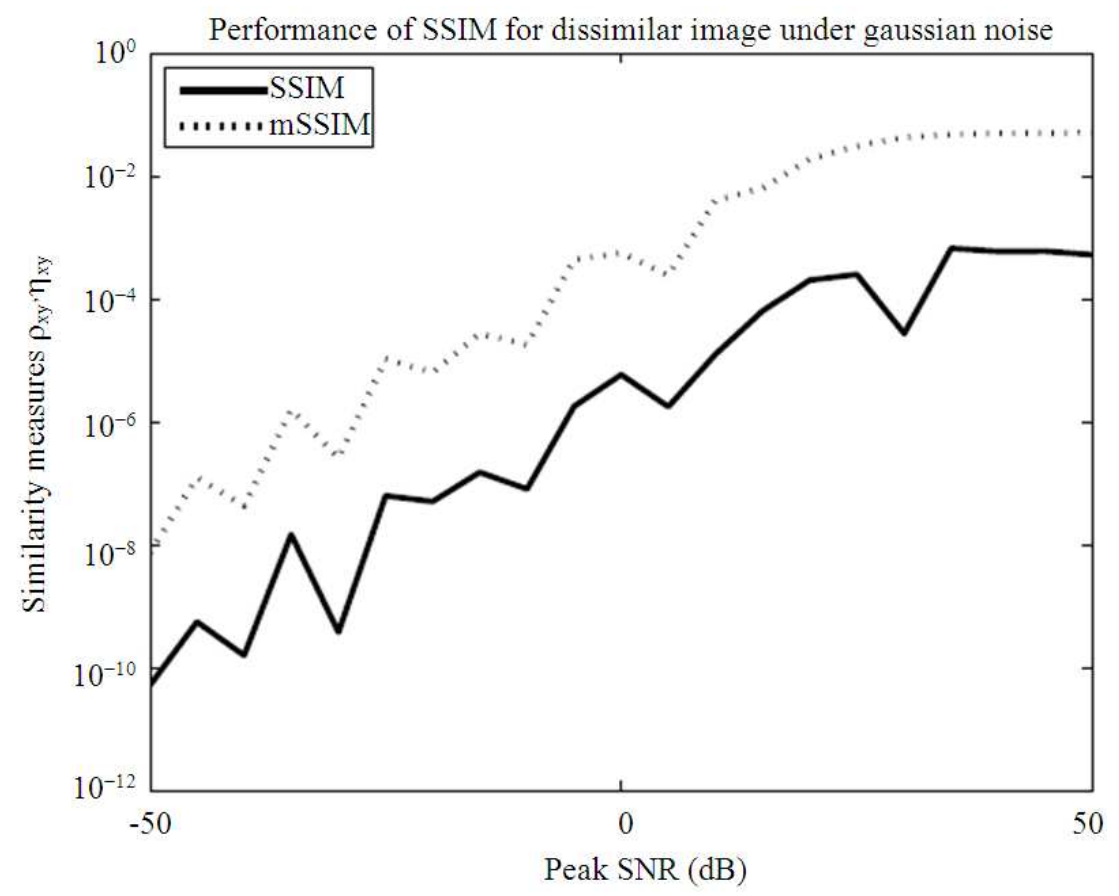

(b)

Fig. 4. Performance of SSIM and eSSIM using dissimilar images under Gaussian noise (a) Above: The test images (b) Below: Performance comparison between SSIM and eSSIM. Logarithmic scale is used 


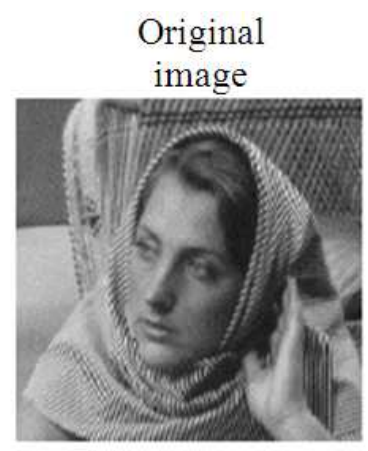

Blurred image; wine length $=16$

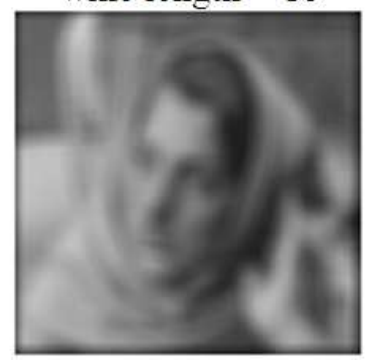

Blurred image; wine length $=3$

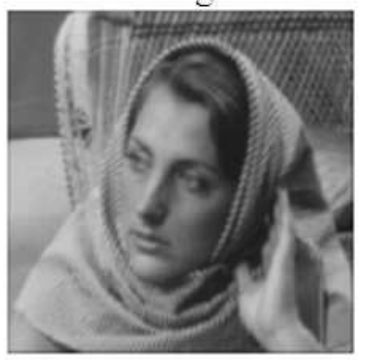

Blurred image;

wine length $=32$

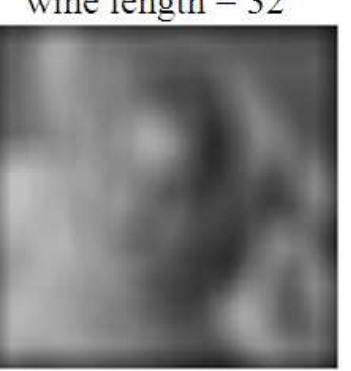

(a)

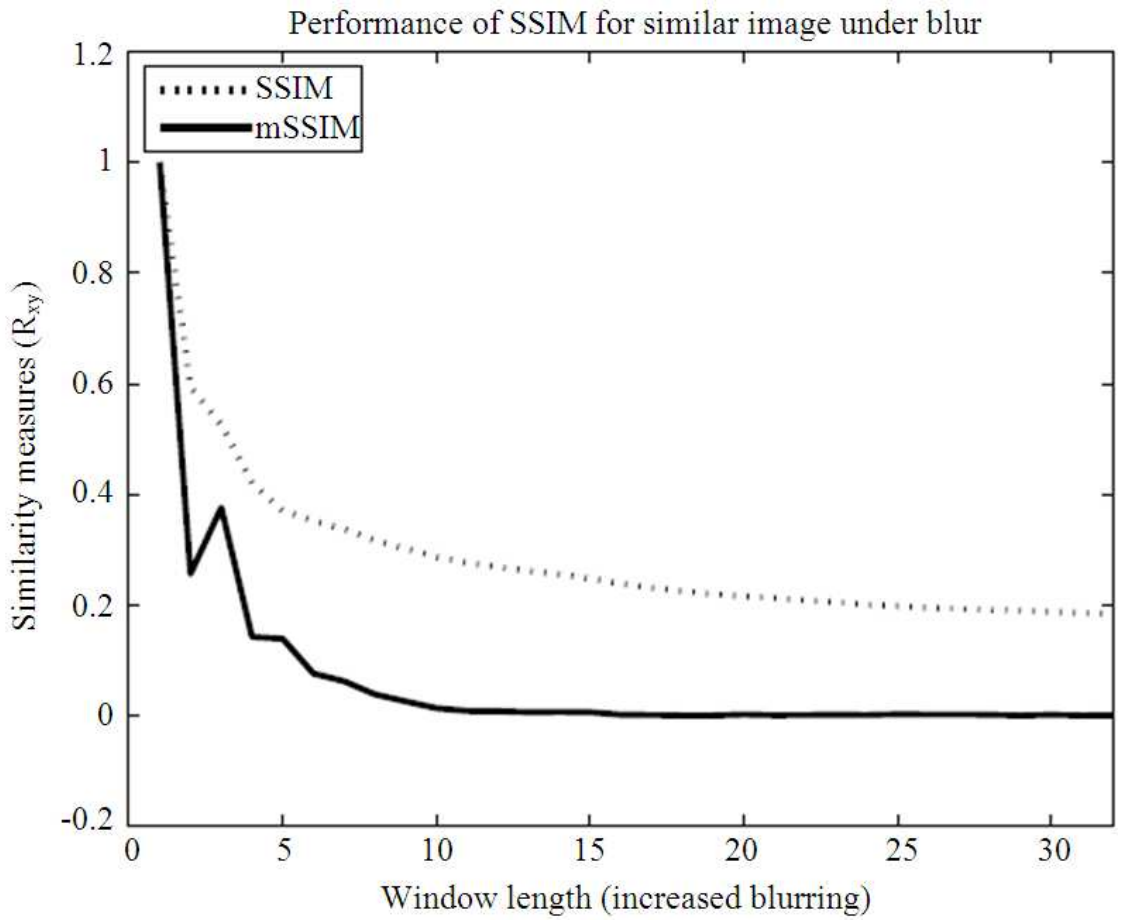

(b)

Fig. 5. Performance of SSIM and eSSIM using similar images under blur (a) Above: The test images (b) Below: Performance comparison between SSIM and eSSIM 


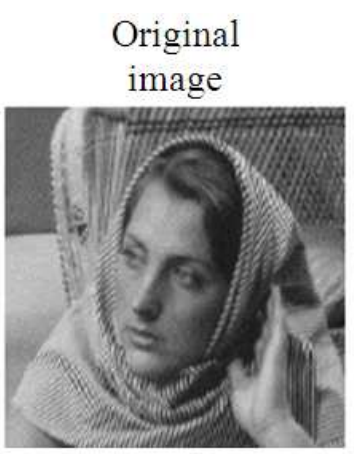

Blurred image; wine length $=16$

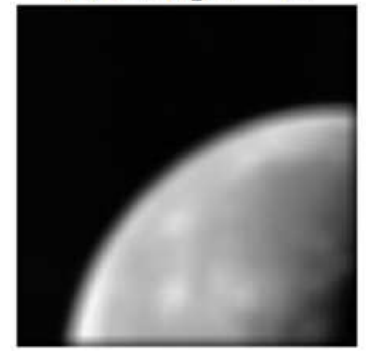

Blurred image;

wine length $=3$

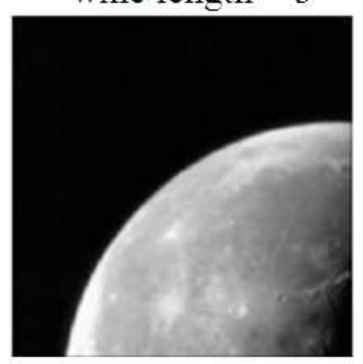

Blurred image;

wine length $=32$

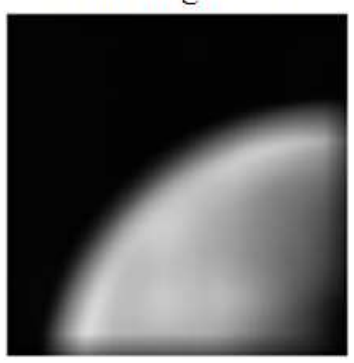

(a)

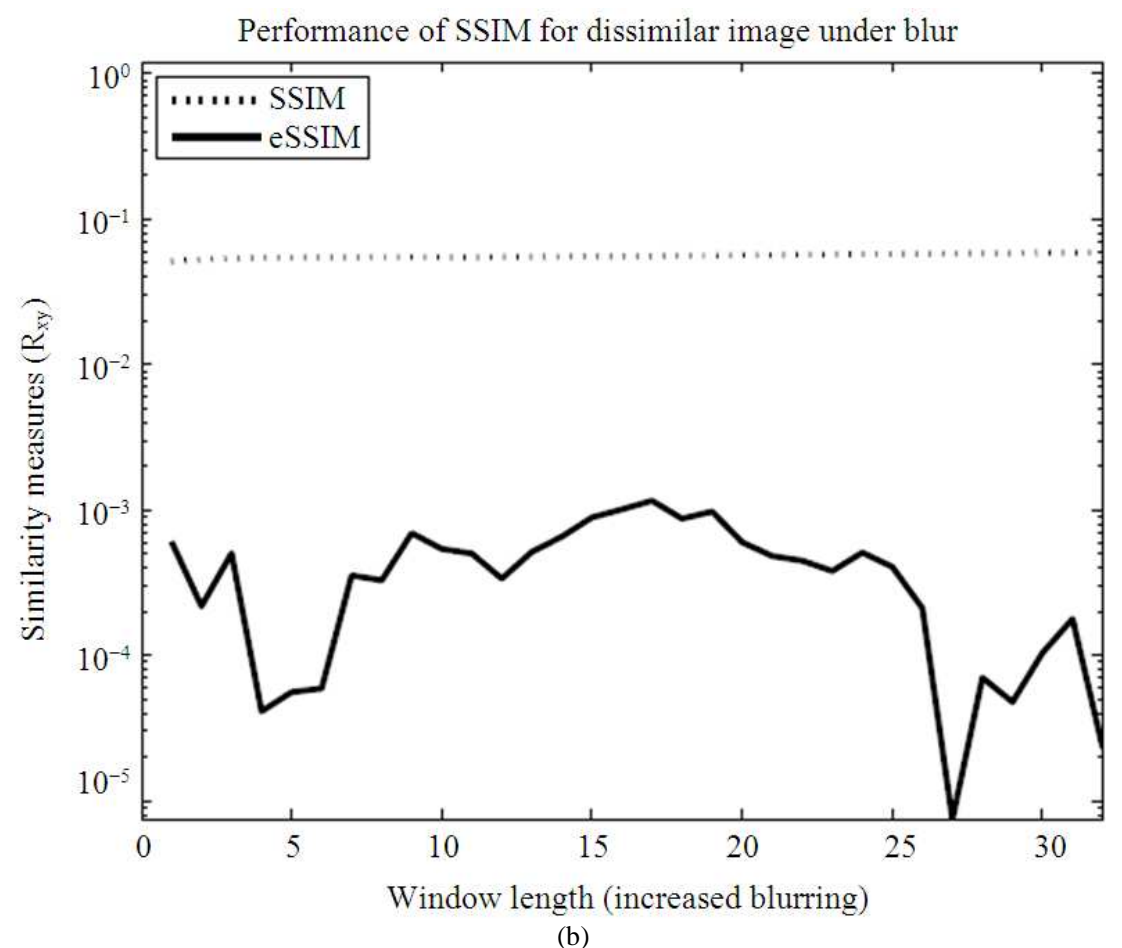

Fig. 6. Performance of SSIM and eSSIM using dissimilar images under blur (a) Above: The test images (b) Below: Performance comparison between SSIM and eSSIM (using logarithmic scale) 


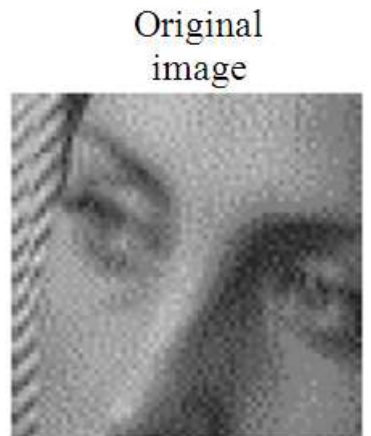

$\lambda=50$; PSNR

$(\mathrm{dB})=10$

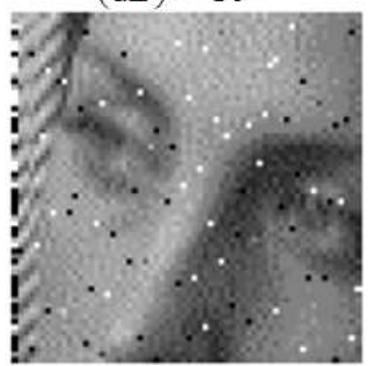

(a)

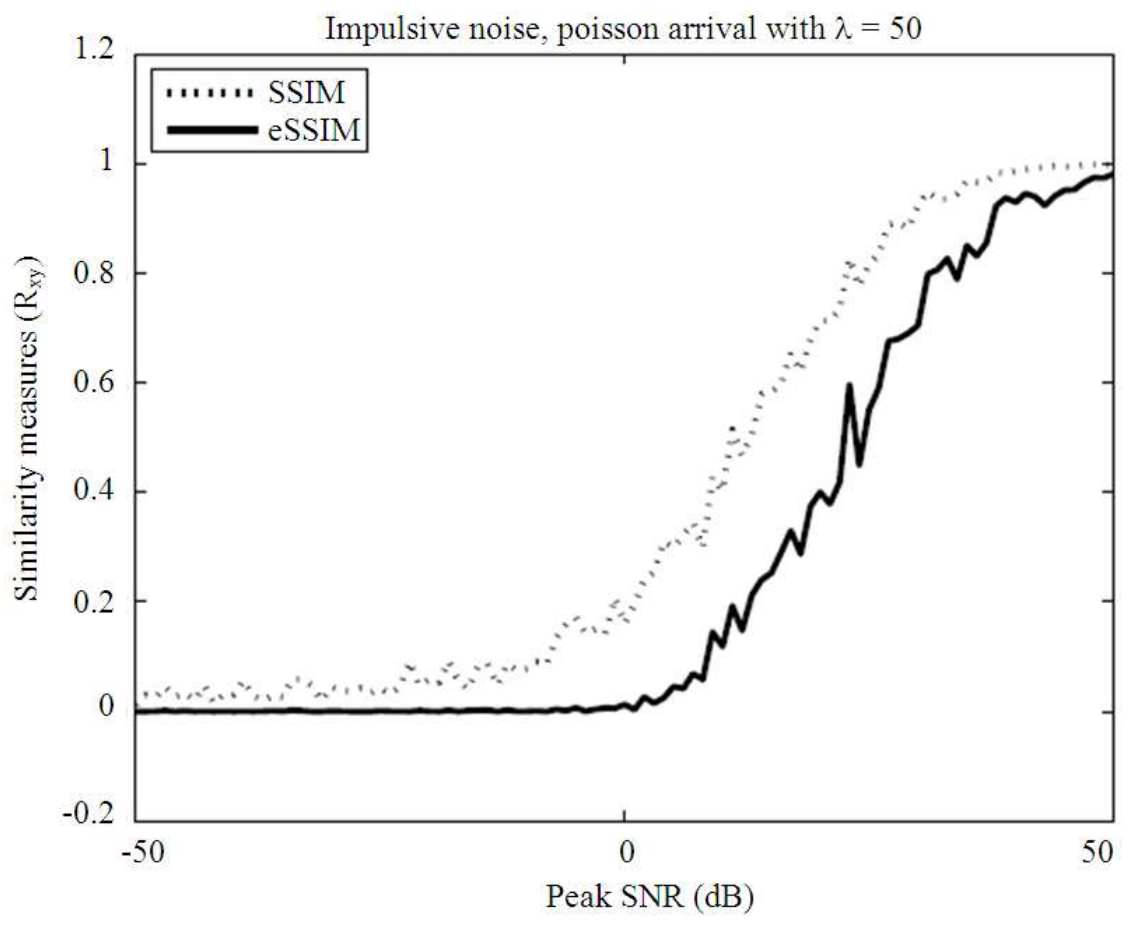

(b)

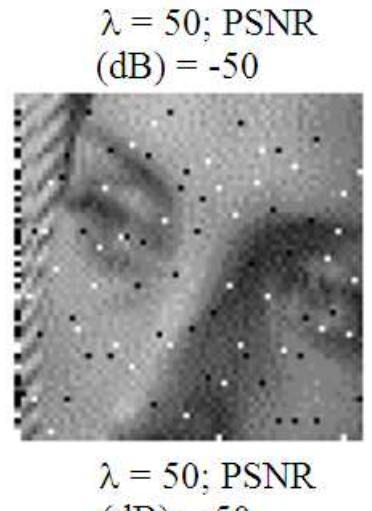

$(\mathrm{dB})=50$

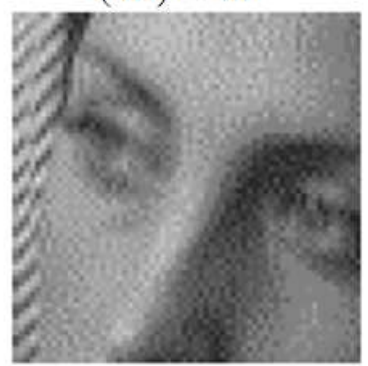




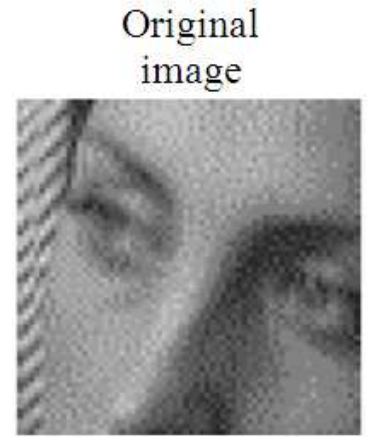

$$
\lambda=50 ; \text { PSNR }
$$$$
(\mathrm{dB})=10
$$

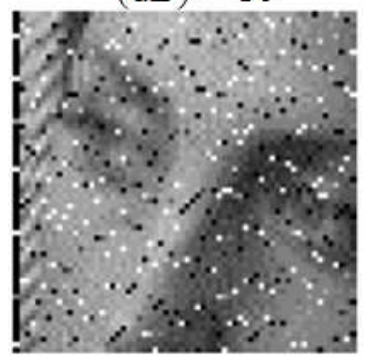

$$
\lambda=50 ; \text { PSNR }
$$

$(\mathrm{dB})=-50$

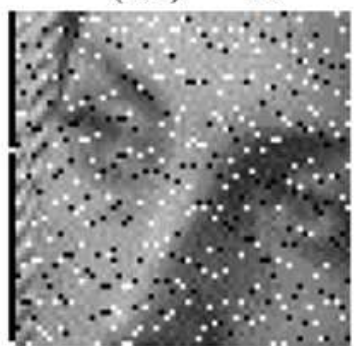

$$
\lambda=50 ; \text { PSNR }
$$

$(\mathrm{dB})=50$

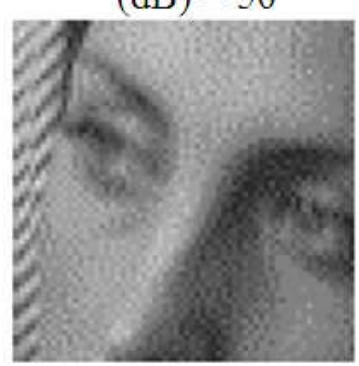

(a)

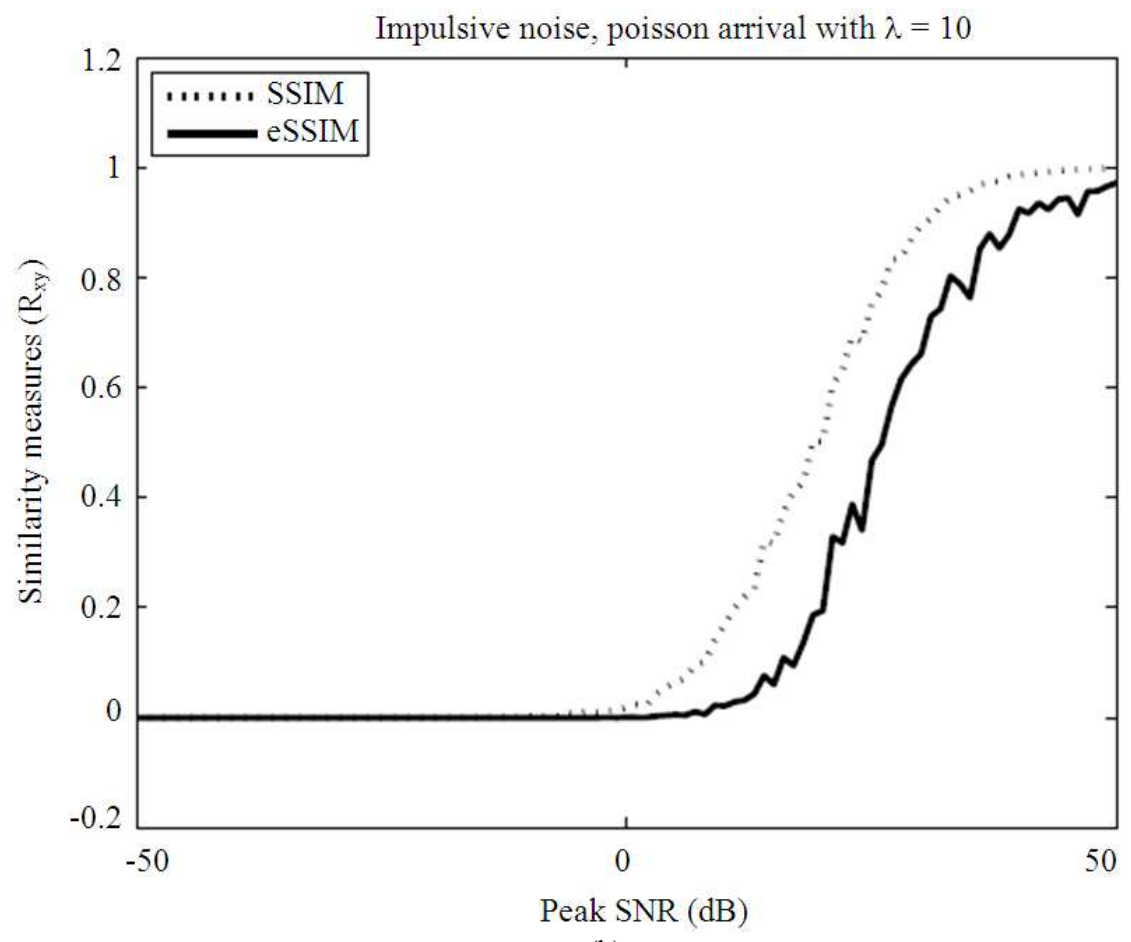

(b)

Fig. 8. Performance of SSIM and eSSIM using dissimilar images under impulse noise with high arrival rate $\lambda=10$ (a) Above: The test images (b) Below: Performance comparison between SSIM and ESSIM 


\subsection{Performance under Impulse Noise}

Impulse noise has been simulated as per Equation 59. Performance of SSIM and the proposed measures have been compared under impulse noise as shown in Fig. 7 and 8 for different values of Poisson parameter $\lambda$.

\section{DISCUSSION}

The conventional SSIM, published by Wang and Bovik (2002), outperforms mSSIM or eSSIM in discovering similarity between similar images, where it gives higher correlation coefficient at similar SNR and blur. Hence, SSIM outperforms the proposed measures in case of comparing two similar images, where it gives reasonable similarity at lower PSNR's than those thresholds of our proposed measures. The reason is that similarity is diluted by using edges or segmentation, which are the bases of our approach. However, SSIM can be misleading for dissimilar images, where mSSIM and eSSIM give almost zero correlation between un-related images.

\section{CONCLUSION}

Two new image-dependent quality assessment measures have been proposed and tested versus structural Similarity Measure (SSIM) under noise (Gaussian and impulsive) and blur. It is shown that the proposed measures can rid SSIM from the disadvantage of giving non-zero correlation between dissimilar images, while SSIM still outperforms the proposed measures in case of comparing two similar images, where it gives reasonable similarity at lower Peak Signal-to- Noise Ratios (PSNR's) than those thresholds of our proposed measures. Little are the works that utilized the capabilities of SSIM for face recognition. As a future direction, we are currently working on using SSIM as a tool for face recognition, where initial results are promising. Also, an extension towards facial expression recognition as per (Lajevardi and Hussain, $2012 ; 2009)$ is under consideration.

\section{REFERENCES}

Al-Mawali, K.S., F.S. Al-Qahtani and Z.M. Hussain, 2010. Adaptive power loading for OFDM-based power line communications impaired by impulsive noise. Proceedings of the IEEE International Symposium on Power Line Communications and Its Applications, Mar. 28-31, IEEE Xplore Press, Rio de Janeiro, pp: 178-182. DOI: 10.1109/ISPLC.2010.5479924
Blasch, E., X. Li, G. Chen and W. Li, 2008. Image quality assessment for performance evaluation of image fusion. Proceedings of the 11th International Conference on Information Fusion, Jun. 30-Jul. 3, IEEE Xplore Press, Cologne, pp: 1-6.

Canny, J., 1986. A Computational approach to edge detection. IEEE Trans. Patt. Anal. Mach. Intell., 8: 679-698. DOI: 10.1109/TPAMI.1986.4767851

Dan, L., D.Y. Bi and Y. Wang, 2010. Image quality assessment based on DCT and structural similarity. Proceedings of the 6th International Conference on Wireless Communications Networking and Mobile Computing, Sept. 23-25, IEEE Xplore Press, Chengdu, pp: 1-4. DOI: 10.1109/WICOM.2010.5600663

Kaur, A., L. Kaur and S. Gupta, 2012. Image recognition using coefficient of correlation and structural similarity index in uncontrolled environment. Int. J. Comput. Appli., 59: 32-39. DOI: 10.5120/9546-3999

Lajevardi, S.M. and Z.M. Hussain, 2009. Facial expression recognition using log-Gabor filters and local binary pattern operators. Proceedings of the International Conference on Communication, Computer and Power, Feb. 15-18, Muscat, Oman, pp: 349-353.

Lajevardi, S.M. and Z.M. Hussain, 2010a. Higher order orthogonal moments for invariant facial expression recognition. Digital Signal Proc., 20: 1771-1779. DOI: 10.1016/j.dsp.2010.03.004

Lajevardi, S.M. and Z.M. Hussain, 2010b. Novel higherorder local autocorrelation-like feature extraction methodology for facial expression recognition. IET Image Processing, DOI: 10.1049/iet-ipr.2009.0100

Lajevardi, S.M. and Z.M. Hussain, 2012. Automatic facial expression recognition: Feature extraction and selection. Signal, Image Video Proc., 6: 159- 169. DOI: 10.1007/s11760-010-0177-5

Li, Z.G., S.L. Xie, W. Yao and S. Rahardja, 2010. A similarity index in the complex wavelet domain. Proceedings of the IEEE Conference on Industrial Electronics and Applications, Jun. 15-17, IEEE Xplore Press, Taichung, pp: 1644-1649. DOI: 10.1109/ICIEA.2010.5515173

Liu, J. and H. Wang, 2011. Image quality assessment based on structure and edge similarity. Proceedings of the International Conference on Intelligent Computation Technology and Automation, Mar. 2829, IEEE Xplore Press, Shenzhen, Guangdong, pp: 1105-1108. DOI: 10.1109/ICICTA.2011.562 
Liu, J., H. Wang and W. Qin, 2011. A new fusion image quality assessment based on edge and structure similarity. Proceedings of the IEEE International Conference on Cyber Technology in Automation, Control and Intelligent Systems, Mar. 20-23, IEEE Xplore Press, Kunming, pp: 112-115. DOI: 10.1109/CYBER.2011.6011774

Sampat, M.P., Z. Wang, S. Gupta, A.C. Bovik and M.K. Markey, 2009. Complex wavelet structural similarity: A new image similarity index. IEEE Trans. Image Proc., 18: 2385-2401. DOI: 10.1109/TIP.2009.2025923

Sheikh, H.R., M.F. Sabir and A.C. Bovik, 2006. A statistical evaluation of recent full reference image quality assessment algorithms. IEEE Trans. Image Proc., 15: 3411-3452. DOI: 10.1109/TIP.2006.881959
Szekely, G.J., M.L. Rizzo and N.K. Bakirov, 2007. Measuring and testing dependence by correlation of distances GJ Szkely. Annals Stat., 35: 2769-2794. DOI: 10.1214/009053607000000505

Wang, Z. and A.C. Bovik, 2002. A universal image quality index. IEEE. Signal Process. Lett., 9: 81-84. DOI: $10.1109 / 97.995823$

Wang, Z., A.C. Bovik, H.R. Sheikh and E.P. Simoncelli, 2004. Image quality assessment: From error visibility to structural similarity. IEEE Trans. Image Proc., 13: 600-612. DOI: 10.1109/TIP.2003.819861

Zhang, F., S. Li, L. Ma and K.N. Ngan, 2009. Limitation and challenges of image quality measurement. Chinese University Hong Kong. 